Revue de l'Institut des langues et cultures

d'Europe, Amérique, Afrique, Asie et Australie

18 | 2013

Les frontières dans le monde hispanique

\title{
Tecnología y educación: El humanista tecnólogo. Deconstruyendo la frontera entre las dos culturas
}

Technology and Education: The Humanistic Technologist. Deconstructing the Border between the Two Cultures

\section{Laura Massimino Amoresano}

\section{OpenEdition}

\section{Journals}

Edición electrónica

URL: http://journals.openedition.org/ilcea/2096

DOI: 10.4000/ilcea.2096

ISSN: 2101-0609

Editor

UGA Éditions/Université Grenoble Alpes

Edición impresa

ISBN: 978-2-84310-251-6

ISSN: 1639-6073

Referencia electrónica

Laura Massimino Amoresano, «Tecnología y educación: El humanista tecnólogo. Deconstruyendo la frontera entre las dos culturas », ILCEA [En línea], 18 | 2013, Publicado el 11 julio 2013, consultado el 19 abril 2019. URL : http://journals.openedition.org/ilcea/2096 ; DOI : 10.4000/ilcea.2096

Este documento fue generado automáticamente el 19 abril 2019.

(c) ILCEA 


\title{
Tecnología y educación: El humanista tecnólogo. Deconstruyendo la frontera entre las dos culturas
}

\author{
Technology and Education: The Humanistic Technologist. Deconstructing the \\ Border between the Two Cultures
}

Laura Massimino Amoresano

1 Cuando se habla del concepto «Frontera», comúnmente se entiende como límite físico, cultural, social o ideológico que sirve para marcar las diferencias entre las partes que quedan a uno $u$ otro lado de ese límite real, imaginario o abstracto. Es decir, una línea, una barrera, un muro, que divide; que determina la posición o pertenencia de unos y otros. En ocasiones, las diferencias entre ambos lados son tan grandes que generan brechas, desniveles.

2 Las causas de estas diferencias pueden venir propiciadas por factores económicos, políticos, educativos, tecnológicos, por citar algunos. Pero estos factores no son actores independientes, autónomos, sino que ejercen una influencia mutua y recíproca; se potencian o limitan, ocasionando que las brechas se amplíen o reduzcan o, incluso, que los límites puedan ser traspasados, desplazando o borrando temporal o permanentemente las fronteras.

3 La revolución tecnológica que venimos experimentando en el último siglo produjo un desarrollo económico que, a su vez, transformó las sociedades actuales, y con ellas las necesidades formativas y educacionales de los ciudadanos. Para poder hacer frente a estas nuevas necesidades es fundamental que algunas de esas líneas fronterizas no sean continuas; que puedan comunicarse ambos lados a través de un diálogo integrador.

4 Vivimos en una sociedad tecnológica. Los jóvenes actuales se mueven a la perfección en Internet y las redes sociales; demuestran grandes habilidades en los videojuegos; emplean programas ofimáticos como procesadores de texto, hojas de cálculo, presentación de 
diapositivas; están conectados a través del ordenador o dispositivos móviles (smartphones o tablets), con los cuales escuchan música, chatean, se comunican, toman fotografías, graban videos o archivos de audio. Estos jóvenes nacieron con la tecnología y su aprendizaje les resulta algo natural porque forma parte de sus vidas. Es lo que Marc Prensky definió como «nativos digitales» ${ }^{1}$.

5 Si bien en Europa se está realizando un gran esfuerzo para integrar las tecnologías de la Información y Comunicación (TIC) en el aula, aún existe una amplia brecha entre la educación no formal, donde las TIC cobran gran protagonismo, y la educación formal o académica. ¿Los motivos? Son numerosos y variados. Algunos derivados de políticas educativas erróneas en materia de formación docente; otros relacionados con la falta de recursos financieros de los centros educativos; y otros enraizados en concepciones tecnofóbicas.

6 Podríamos debatir ampliamente sobre las ventajas y contras del uso de la tecnología en los procesos de enseñanza/aprendizaje. Sin embargo, como paso previo a ese debate, es necesario comprender el contexto actual en el cual se desarrolla la educación formal.

7 La revolución tecnológica que produjo una transformación económica y social en Europa dejó en evidencia dos cuestiones fundamentales. Por un lado, que los sistemas educativos eran anacrónicos. Ken Robinson lo definió muy bien: «Se crearon en el pasado, en una época distinta para responder a retos diferentes $»^{2}$. Y, por otro, la necesidad de una reforma urgente en el ámbito educativo.

8 ¿El problema? Las sociedades actuales no son las mismas que las del siglo XIX o las de principios y mediados del siglo xx. El mundo se había transformado económica, social y culturalmente en los últimos 50 años. La revolución tecnológica surgida a partir de las Tecnologías de la Información y Comunicación establecieron los fundamentos de una nueva economía y sociedad llamada del conocimiento.

9 Es recién a partir de 1999, con la Declaración de Boloña ${ }^{3}$, cuando se inicia el proceso de convergencia del Espacio Europeo de Educación Superior, cuya culminación será la reforma educativa llevada a cabo en 2010 con la implementación del Plan Boloña.

Estamos siendo testigos de una concienciación creciente en la mayor parte del mundo académico político, y en la opinión pública, de la necesidad de establecer una Europa más completa y de mayor alcance construida, en particular, mediante el desarrollo y fortalecimiento de sus dimensiones intelectual, cultural, social y científica y tecnológica [...].

En la actualidad, la Europa del conocimiento está ampliamente reconocida como un factor irremplazable para el crecimiento social y humano y es un componente indispensable para consolidar y enriquecer a la ciudadanía Europea, capaz de dar a sus ciudadanos las competencias necesarias para afrontar los retos del nuevo milenio, junto con una conciencia de compartición de valores y pertenencia a un espacio social y cultural común ${ }^{4}$.

10 Con anterioridad al inicio del Proceso de Boloña, en 1985, el historiador Melvin Kranzberg, con una visión casi oracular, predecía que las

aplicaciones derivas de las TIC estaban generando un conjunto de nuevos bienes y servicios que extendían un amplio abanico de conexiones hacia el resto de actividades económicas. Esta nueva manera de invertir, producir y consumir iría unida a nuevos patrones de comportamiento de todos los agentes económicos, que desembocarían en cambios sociales, institucionales y culturales de primera magnitud 5 . 
11 Sin embargo, los sistemas educativos se habían forjado bajo la Revolución Industrial y, por lo tanto, tras la sombra de un modelo basado en la producción masiva. En las primeras escuelas los profesores poseían unos conocimientos que transmitían o dictaban a los alumnos, quienes los memorizaban y luego repetían en un examen. Fue en el siglo XIX cuando comenzaron a regularse los sistemas nacionales educativos. «Un proceso estandarizado y lineal en el que se enseñaban aquellas materias que se consideraban útiles para la recién nacida economía industrial» ${ }^{6}$. Un sistema diseñado para garantizar que todos los alumnos consiguieran los mismos objetivos académicos y adquirieran los mismos aprendizajes a través de un único método de enseñanza; aprendizajes que debían ser mesurables mediante procesos estandarizados de evaluación.

Los avances tecnológicos producidos a partir de la segunda mitad del siglo xx permitieron profundizar en campos científicos hasta el momento limitados, como el de la neurociencia. Desde hace décadas contamos con abundante información, procedentes de diversas investigaciones y estudios científicos, sobre los procesos de aprendizaje y el funcionamiento del cerebro que nos han permitido conocer cuáles son los mecanismos naturales del aprendizaje, como la experimentación, la reflexión, la importancia de las emociones y la motivación y, sobre todo, que aprendemos haciendo, no memorizando, y no todos aprendemos de la misma manera.

13 Esta realidad de la economía y sociedad del conocimiento, y la consiguiente necesidad de adaptar los sistemas educativos con el fin de preparar a los ciudadanos del futuro, se aprecia claramente en los diversos comunicados y declaraciones del Proceso de Boloña:

La educación superior debería jugar un papel esencial en la promoción de la cohesión social, en la reducción de las desigualdades y en la elevación del nivel del conocimiento, destrezas y competencias en el seno de la sociedad. Las políticas de educación superior deberían enfocarse a maximizar el potencial de las personas en cuanto a su desarrollo personal y su contribución a una sociedad sostenible, democrática y basada en el conocimiento ${ }^{7}$.

El aprendizaje para toda la vida es un elemento esencial del Área de Educación Superior Europea. En la Europa futura, construida sobre una sociedad y economía basadas en el conocimiento, las estrategias del aprendizaje de toda la vida son necesarias para encarar los desafíos de la competitividad y el uso de nuevas tecnologías y para mejorar la cohesión social, la igualdad de oportunidades y la calidad de vida ${ }^{8}$.

14 En 1994, la Comisión Internacional sobre Educación para el siglo XXI, presidida por Jacques Delors, presentó para la UNESCO el informe La educación encierra un tesoro, en el cual participaron especialistas de todo el mundo. En él se presentaban los cuatro pilares de la educación, donde queda reflejada la «necesidad de trabajar en valores y competencias para conseguir un aprendizaje a lo largo de la vida» ${ }^{9}$. Estos cuatro pilares son:

aprender a conocer;

aprender a hacer;

aprender a convivir; aprender a ser.

15 En definitiva, se plantea desarrollar competencias para la compresión; la capacidad de aprender a aprender. Se busca el desarrollo integral de la persona a través de la imaginación, la creatividad y el pensamiento crítico, que permita el conocimiento de uno mismo y del otro, superando las fronteras del individualismo, donde la gran beneficiada sea la sociedad. 

disposiciones naturales de cada individuo $\mathrm{y}$, por otra, a resultados de experiencias y aprendizajes pasados, diferentes según los contextos y las culturas. Estos autores proponen un camino para mejorar el aprendizaje por medio de la reflexión personal y de las peculiaridades diferenciales en el modo de aprender. 

propone un modelo de enseñanza centrado en el aprendizaje del alumno, quien es responsable y partícipe de este proceso. Ello implica emplear estrategias didácticas donde el estudiante tenga que enfrentarse a situaciones reales y significativas para él, aplicar los conocimientos adquiridos para la búsqueda de soluciones, tomar decisiones y aprender de forma autónoma, reflexiva y crítica, con el fin de construir aprendizajes significativos. En consecuencia, se produce también un cambio en la función del profesor, si cabe aún más importante que la de mero trasmisor de información, al convertirse en guía durante el proceso de aprendizaje, ayudando a los alumnos a acceder y crear conocimiento, poniendo en juego sus estilos e inteligencias.

32 En este nuevo modelo educativo lo que prima, lo principal, es el proceso, el camino que se recorre desde la información al conocimiento y a la aplicación del mismo, no el resultado o la realización de una prueba estandariza como son los exámenes.

Cestán generando una nueva concepción de la adquisición del conocimiento. Ya no es necesario que nuestro cerebro almacene todos los datos porque los ordenadores lo hacen por nosotros. Lo importante en este nuevo contexto es saber formular y plantear las preguntas correctas en relación a esos datos, dar forma a un pensamiento creativo, combinando diversas perspectivas y aproximaciones a un tema, saber trabajar con la información, aprender a discernir entre lo que es válido y no. 
humanistas analizamos la influencia que están teniendo en la sociedad, la importancia de la formación técnico humanista y las nuevas disciplinas que están surgiendo a partir de ellas. Un ejemplo es la filología digital, que intenta comprender la implicación del ordenador en la literatura a través de la teoría del hipertexto y su empleo para la producción literaria digital.

países angloamericanos, de la relación entre las Humanidades y la Informática surgió la disciplina Humanities Computing (Humanidades Digitales) ${ }^{14}$, que estudia los nuevos métodos surgidos a partir de las TIC en las Humanidades y, sobre todo, reflexiona sobre su uso, adentrándose en cuestiones filosóficas y en el desarrollo del pensamiento crítico. Es decir, en el «cómo» y el «qué» del uso de las Nuevas Tecnologías, que modifican la manera en que se entiende, genera y distribuye el conocimiento ${ }^{15}$.

Ejemplo de integración de las TIC en aula como recurso que facilita el proceso de aprendizaje del alumno dentro de la nueva concepción que propone el Plan Boloña es el proyecto educativo Archivo de la Frontera ${ }^{16}$ : Una Comunidad Virtual Histórica y un Banco de recursos o contenidos.

Una Comunidad Virtual es una agrupación de personas que, contando con unos intereses comunes sobre temas específicos - en este caso la Historia-colaboran e interaccionan en un espacio virtual concreto, como es la plataforma en Internet. Por lo tanto, es un lugar que permite la participación y el intercambio de información, ideas y experiencias, a la vez que superar las limitaciones geográficas y temporales en los trabajos o investigaciones colaborativas.

41 La idea inicial del proyecto fue la construcción compartida del conocimiento mediante la creación de un banco de contenidos digitales, que permitiera la difusión del patrimonio histórico y el acceso a dicha información. El objetivo es poner a disposición de estudiantes, profesores e investigadores materiales y documentos históricos que puedan contribuir a sus investigaciones y estudios. Investigaciones que posteriormente se publican en la plataforma convirtiéndose en un nuevo material o recurso de trabajo para otros investigadores, tejiendo una red de conocimiento cada vez más amplia y generando así esa construcción compartida que mencionaba. 

destacan: disciplina.

Los textos presentados son fuentes manuscritas o impresas, completas o en fragmentos significativos, bien inéditas, procedentes de Archivos y Bibliotecas, o bien de grandes repertorios documentales impresos. Lesuarios registrados tienen la opción de comentar los documentos y responder a otros comentarios publicados. Ello permite la interacción entre usuarios que estén trabajando temáticas similares mediante la aportación de información complementaria o consultas.

Desde la creación del Archivo de la Frontera, se empleó la documentación publicada como material de trabajo en las clases dictabas por el Dr. Sola ${ }^{17}$ en la Universidad de Alcalá. Los alumnos contaban con la posibilidad de publicar los trabajos realizador durante el curso en la plataforma online.

5 Esta experiencia nos permitió comprobar que los estudiantes, al trabajar directamente con los documentos históricos y las fuentes primarias, y enfrentarse a situaciones reales de investigación con una finalidad clara para ellos, en las cuales tenían que aplicar los conocimientos teóricos adquiridos en las clases, buscar soluciones y tomar decisiones, conseguía mejores resultados de aprendizaje.

6 Poco tiempo después, la ANECA (Agencia Nacional de Evaluación de la Calidad y Acreditación española) publicaba las nuevas competencias, destrezas y habilidades que los alumnos debían adquirir para el grado de historia según el Plan Boloña. Entre las que

capacidad de gestión de la información;

capacidad de leer textos historiográficos o documentos originales, así como de transcribir, resumir y catalogar información de forma pertinente;

conocimiento y habilidad para usar los instrumentos de recopilación de información y las técnicas necesarias para estudiar documentos de determinados períodos;

capacidad de identificar y utilizar fuentes de información para la investigación histórica; capacidad para manejar los recursos y técnicas informáticas y de Internet.

7 Y entre los objetivos del Grado de Historia se indicaba que debía procurar un conocimiento racional y crítico del pasado; proporcionar un conocimiento básico de los métodos, técnicas e instrumentos de análisis del historiador; y que los alumnos debían saber expresarse con claridad y coherencia empleando la terminología de la propia

8 Diversos informes e investigaciones realizados por la propia ANECA y el Grupo de Historia del Tuning Project ${ }^{18}$ revelaban la necesidad de fortalecer los vínculos entre teoría y práctica investigadora, así como mejorar la enseñanza en el manejo de instrumentos de análisis, y sugerían la idoneidad de pasar de un modelo pedagógico de transmisión del conocimiento a otro que enfatice en andamiaje de los procesos.

La experiencia con la que contábamos, los cambios que el Plan Boloña planteaba en relación al currículo de Grado, los objetivos que se buscaban y las competencias que debían desarrollar los alumnos, nos llevó a plantear una metodología didáctica que introdujera a los estudiantes en los métodos y técnicas de investigación histórica, ayudándonos para ello de las TIC. Así nació el Juego del Legajo, una metodología didáctica que propone al alumno el contacto directo con las fuentes primarias y la superación, con ayuda del profesor, de los problemas inherentes a la investigación.

50 El objetivo es partir de un documento histórico original y reelaborarlo hasta que pueda evocar o narrar una historia. La finalidad del Juego es que el alumno se familiarice con la 
profesión de historiador, que desarrollen la capacidad de crear conocimiento y exponer sus ideas, análisis y conclusiones.

51 Todos los documentos - en este caso contamos con el Legajo 486 y 487 del Archivo General de Simancas - están digitalizados y los estudiantes eligen una o varias piezas documentales para el abordaje en grupo. En las clases iniciales se les da la información necesaria para su desarrollo, que si está bien resuelto se convertirá en un contenido del Archivo de la frontera. Se trabajaba conjuntamente con los alumnos en las glosas, transcripciones y comentarios de los documentos, además de la visualización de algunos documentales para situarlos en el contexto del programa.

aforma online no sólo es una base de datos donde los alumnos buscan información para sus investigaciones, también facilita una serie de herramientas de comunicación y colaboración, como la creación de grupos de trabajo. Al inicio del curso se crea un grupo de la asignatura y se invita a participar a los estudiantes. Estos grupos permiten una comunicación más personalizada entre alumnos y profesores, facilitan un espacio para las consultas, la organización de las clases y prácticas: los alumnos pueden compartir recursos interesantes para la asignatura y coordinarse para organizar el simposio donde presentan las investigaciones; el profesor, por su parte, puede publicar materiales de trabajo, textos, realizar un seguimiento de las prácticas y comentar con los alumnos diversas cuestiones.

Dos son los factores clave del Juego. Por un lado su libertad de enfoque, que permite a los estudiantes seleccionar aquellas actividades que más se adapte a sus intereses y capacidades. Por otro, el formato de presentación de la investigación realizada, que pueden variar desde trabajos más académicos a presentaciones tipo comic o piezas audiovisuales.

En todos los casos, para elaborar el producto final, los alumnos parten de fuentes primarias y documentos publicados en el Archivo de la Frontera, para lo cual deben comenzar con la transcripción paleográfica, la contextualización de los hechos que se narran y/o el período histórico en el que transcurre, así como de los personajes presentes en la narración. A partir de ahí, poner en juego su creatividad y capacidad de análisis y síntesis para elaborar el producto final.

Todo junto llevará a una versión lo más correcta posible de la pieza documental. Y de dicha pieza, a su vez, pueden surgir posibles variantes, generadas en distintas ediciones y niveles del juego, hasta conseguir un recurso o documento definitivo.

La práctica finaliza con un simposio o congreso organizado por los estudiantes, donde exponen y presentan sus investigaciones ante sus compañeros y el profesor, con el fin de adquirir soltura en la transmisión del conocimiento y la exposición oral del mismo.

Otro factor importante en los procesos de aprendizaje es la motivación. En el caso del Juego, los estudiantes, al contar el Archivo de la Frontera con ISBN, tienen la oportunidad de que sus investigaciones sean valoradas como publicación científica. Si bien ello supone un gran incentivo, la experiencia nos ha demostrado que un factor motivacional mayor es que el alumno, a través de la práctica, se siente partícipe en la construcción de la Historia. He presentado un ejemplo de cómo pueden ayudarnos las TIC a desarrollar destrezas y competencias necesarias para el desempeño profesional en la Sociedad del Conocimiento. Sin embargo, no podemos obviar que las Tecnologías de la Información y la Comunicación no sólo han generado cambios en nuestras sociedades a nivel económico, laboral y educativo, sino también en la manera en la que conocemos el mundo e interactuamos en 
él, en nuestra concepción misma del conocimiento y cómo se distribuye en la sociedad, y en la manera en que interpretamos las realidades existentes y nos comunicamos con los otros.

El punto de referencia constante para el Humanismo es el ser humano, en todas sus dimensiones. El hombre como ser cargado de discursos científicos, filosóficos, literarios, políticos, históricos, sociales. El hombre como ser pensante y creador, pero también como ser social y emocional. Conocer y comprender al hombre significa también estudiar sus manifestaciones artísticas y culturales; sus valores e ideales; su entorno. En definitiva, lo que se busca es la comprensión y explicación del mundo y del ser en el mundo.

61 Como humanistas, al estudiar el pasado nos damos cuenta que no sólo han cambiado las formas de producción y comunicación. Los avances científicos y tecnológicos también han propiciado un cambio en la concepción que tenemos sobre nosotros mismos como seres humanos en el mundo. Claro ejemplo de este cambio fueron los descubrimientos y teorías de Galileo y Copérnico, que suponían que el hombre dejaba de ser el centro del universo, lo cual supuso un terremoto ideológico.

Si analizamos el Humanismos renacentista descubriremos que uno de los factores clave para su difusión y alcance fue otro avance tecnológico: la invención de la imprenta permitió el acceso a las ideas y su distribución. La gente podía leerlas por sí misma, sin depender de una minoría culta del clero, y ello generó también nuevas posibilidades intelectuales y grandes cambios cualitativos.

En el Renacimiento, las Humanidades caminaban a la par del desarrollo científico tecnológico y ejercían una influencia intelectual recíproca. Con la Ilustración y el academicismo se parceló el conocimiento. El Romanticismo y el Positivismo supusieron su completa ruptura.

Las Ciencias y las Humanidades, antaño unidas, quedaron divididas en lo que C. P. Snow definió en 1959 como «las dos Culturas»" ${ }^{19}$. Para él esta ruptura de la comunicación entre ciencia y humanidades suponía uno de los principales inconvenientes para solucionar los problemas mundiales, porque cuando las ciencias y las humanidades se separan ninguna sociedad es capaz de pensar con cordura. Desde entonces, grandes personalidades e investigadores de todos los campos del saber han advertido de los peligros de esta separación.

65 La Historia del Hombre está plagada de hechos que cambiaron nuestra visión del mundo; de lo que somos capaces como seres humanos; de cómo determinados hechos históricos, unidos a los avances tecnológicos - la bomba atómica, por ejemplo-, han influido o modificado las conciencias colectivas. Como bien explica Ruiz Zúñiga ${ }^{20}$,

La naturaleza de la tecnología se comprende especialmente en su función social. [...] Si la tecnología moderna genera destrucción, explotación, alienación y desequilibrio con la naturaleza, es porque ha sido estructurada y usada provocando esas consecuencias por los grupos sociales y naciones dominantes en nuestras sociedades. La tecnología no es una realidad políticamente neutra [...] que en sí misma provoca el bien o el mal, la pasividad, la enajenación o la angustia existencial. Aunque ocupando un papel central en la configuración de la sociedad moderna, la tecnología es un elemento secundario y dependiente frente al conjunto de la sociedad y sus reglas. [...] Al elevar la tecnología a una categoría autónoma (buena o mala) se introducen condiciones para eximir de responsabilidades éticas a los hombres y a los grupos sociales. responsabilidades éticas, que son inseparables del uso que hacemos de la tecnología. Esta 
conciencia ético-política-histórica-social no puede separarse de los avances científicos y tecnológicos, de los cuales somos sus autores materiales e ideológicos.

En definitiva, necesitamos comprender la construcción de este nuevo mundo digital, analizar por qué las TIC tienen tanto impacto cultural y cómo el desarrollo tecnológico afecta a los seres humanos: su forma de pensar, de comunicarse, de expresarse, de percibir el mundo.

En esta sociedad del conocimiento, donde la tecnológica es un factor desencadenante y potenciador -tecnología que evoluciona a un ritmo exponencial, el cual muchas veces no permite que a nivel social puedan asimilarse los cambios antes de que se produzcan otros nuevos-, es absolutamente necesario que deconstruyamos la frontera entre las Dos Culturas - la científica y la humanista- para, al igual que sucedía en el Renacimiento, converjan en la Cultura. Este proceso, desde un punto de vista educativo, debe partir de la inclusión en los programas académicos del desarrollo de conocimientos, competencias, destrezas y habilidades que permitan una formación integral e interdisciplinar de humanistas tecnólogos y científicos humanistas.

\section{BIBLIOGRAFÍA}

ALONSO Catalina, GALLEGo Domingo y Honey Peter, Los Estilos de Aprendizaje: Procedimientos de diagnóstico y mejora, Bilbao, Ediciones Mensajero, 1994.

ANECA, Libro Blanco. Título de Grado en Historia [en línea], Madrid, Agencia Nacional de Evaluación de la Calidad y Acreditación, 2004. Disponible en <www.aneca.es/activin/docs/ libroblanco_jun05_historia.pdf> (consultado el 20/12/2011).

-, Reflex. Informe ejecutivo. El profesional flexible en la Sociedad del Conocimiento [en línea], Madrid, Agencia Nacional de Evaluación de la Calidad y Acreditación, 2007a. Disponible en < www.aneca.es/media/158162/informeejecutivoaneca_jornadasreflexv20.pdf> (consultado el 20/12/2011).

-, Informe sobre el estado de la evaluación de la calidad en las universidades españolas [en línea], Madrid, Agencia Nacional de Evaluación de la Calidad y Acreditación, 2007b. Disponible en < www.aneca.es/media/151208/informe_micinn_071202.pdf> (consultado el 20/12/2011).

COMUNICADO DE LONDRES, Hacia el Espacio Europeo de Educación Superior: respondiendo a los retos de un mundo globalizado [en línea], Vlaams Ministerie van Onderwijs en Vorming, 2007. Disponible en < www.ond.vlaanderen.be/hogeronderwijs/bologna/links/

language/2007_London_Communique_Spanish.pdf>(consultado el 20/12/2011).

DEClARACIÓN DE BOloÑA, El Espacio Europeo de la Enseñanza Superior [en línea], Espacio Europeo de Educación Superior, 1999. Disponible en <http://www.eees.es/pdf/Bolonia_ES.pdf> (consultado el 20/12/2011).

DECLARACIÓN DE PRAGA, Hacia el Área de la Educación Superior Europea [en línea], Espacio Europeo de Educación Superior, 2001. Disponible en <www.eees.es/pdf/Praga_ES.pdf> (consultado el 20/12/2011). 
DELORS Jacques, La Educación encierra un tesoro [en línea], Santillana, Ediciones UNESCO, 1994. Disponible en <www.unesco.org/education/pdf/DELORS_S.PDF> (consultado el 20/12/2011). GARDNER Howard, Inteligencias múltiples. La teoría en la práctica [en línea], Barcelona, Paidos, 1998. Disponible en <http://ict.edu.ar/renovacion/wp-content/uploads/2012/02/ Gardner_inteligencias.pdf> (consultado el 20/12/2011).

LEIBRANDT Isabella, «Humanidades digitales ¿Ciencia ficción o realidad inminente?» [en línea], Espectáculo, n. ${ }^{\circ} 33,2006$. Disponible en <www.ucm.es/info/especulo/numero33/humadigi.html> (consultado el 20/12/2011).

PRENSKY Marc, Nativos e Inmigrantes Digitales [en línea], Cuadernos SEK 2.0, 2001. Disponible en < www.marcprensky.com/writing/Prensky-NATIVOS E INMIGRANTES DIGITALES (SEK).pdf> (consultado el 20/12/2011).

PUNSET Eduard, «El sistema educativo es anacrónico» [en línea], RTVE, Redes, 2011a. Disponible en <www.rtve.es/television/20110304/redes-sistema-educativo-anacronico/413516.shtml> (consultado el 20/12/2011).

-, «De las inteligencias múltiples a la educación personalizada» [en línea], RTVE, Redes, 2011b. Disponible en <www.rtve.es/television/20111209/inteligencias-multiples-educacionpersonalizada/480968.shtml> (consultado el 20/12/2011).

RUIZ ZÚÑIGA Ángel, «Consideraciones histórico-filosóficas sobre la tecnología» [en línea], Revista de Filosofía de la Universidad de Costa Rica, n. ${ }^{\circ}$ 59, 1986, pp. 25-52. Disponible en <http://inif.ucr.ac.cr/ recursos/docs/Revista\%20de\%20Filosof\%C3\%ADa\%20UCR/Vol.\%20XXIV/No.59/Consideraciones \%20Historico-Filosoficos\%20Sobre\%20la\%20Tecnologia.pdf> (consultado el 20/12/2011).

SNOw Charles Percy, The Scientific Revolution [en línea], New York, Cambridge University Press, 1961. Disponible en <http://sciencepolicy.colorado.edu/students/envs_5110/snow_1959.pdf> (consultado el 20/12/2011).

TUBELla i CASADEVAll Imma y VILASECA I REQUena Jordi (coord.), Sociedad del conocimiento. Cómo cambia el mundo ante nuestros ojos, Barcelona, UOC, 2005.

\section{NOTAS}

1. Para mayor información consultar M. Prensky, Nativos e Inmigrantes Digitales [en línea], Cuadernos SEK 2.0, 2001.

2. E. Punset, «El sistema educativo es anacrónico» [en línea], RTVE, Redes, 2011a.

3. Para una información más detallada del Proceso de Boloña puede consultarse la Web: <http:// europa.eu/legislation_summaries/education_training_youth/lifelong_learning/c11088_es.htm>.

4. Declaración de Boloña, El Espacio Europeo de la Enseñanza Superior [en línea], Espacio Europeo de Educación Superior, 1999.

5. Citado en I. Tubella i Casadevall y J. Vilaseca i Requena (coord.), Sociedad del conocimiento. Cómo cambia el mundo ante nuestros ojos, Barcelona, UOC, 2005, p. 39.

6. E. Punset, «El sistema educativo es anacrónico», op. cit.

7. Comunicado de Londres, Hacia el Espacio Europeo de Educación Superior: respondiendo a los retos de un mundo globalizado [en línea], Vlaams Ministerie van Onderwijs en Vorming, 2007.

8. Declaración de Praga, Hacia el Área de la Educación Superior Europea [en línea], Espacio Europeo de Educación Superior, 2001.

9. J. Delors, La Educación encierra un tesoro [en línea], Santillana, Ediciones UNESCO, 1994, p. 34. 
10. C. Alonso, D. Gallego y P. Honey, Los Estilos de Aprendizaje: Procedimientos de diagnóstico y mejora, Bilbao, Ediciones Mensajero, 1994, p. 48.

11. P. Honey (ibid.) describe sus Estilos de Aprendizaje de esta forma:

-Activos: se implican plenamente y sin prejuicios en nuevas experiencias. Son de mente abierta, nada escépticos y acometen con entusiasmo las tareas nuevas. Son personas muy de grupo que se involucran en los asuntos de los demás.

-Reflexivos: les gusta considerar las experiencias y observarlas desde diferentes perspectivas. Recogen datos, analizándolos con detenimiento antes de llegar a alguna conclusión.

-Teóricos: adaptan e integran las observaciones dentro de teorías lógicas y complejas. Tienden a ser perfeccionistas. Les gusta analizar y sintetizar y son profundos en su sistema de pensamiento. -Pragmáticos: descubren el aspecto positivo de las nuevas ideas y aprovechan la primera oportunidad para experimentarlas. Les gusta actuar rápidamente y con seguridad con aquellos proyectos que les atraen.

12. Para más información consultas H. Gardner, Inteligencias múltiples. La teoría en la práctica [en línea], Barcelona, Paidos, 1998.

13. E. Punset, «De las inteligencias múltiples a la educación personalizada» [en línea], RTVE, Redes, 2011b.

14. Para una información más detallada puede consultarse I. Leibrandt, «Humanidades digitales ¿Ciencia ficción o realidad inminente?» [en línea], Espectáculo, n. ${ }^{\circ}$ 33, 2006.

15. Las TIC permiten la creación de contenido hipermedial: integrar texto, imagen, sonido, vídeo y animaciones. La combinación de estos elementos brinda al alumno distintos tipos de información, que al combinarse ofrecen una experiencia de aprendizaje más amplia y profunda. Otra característica es la hipertextualidad de la información. Los textos se combinan entre sí mediante un sistema de enlaces de navegación que permiten profundizar en el contenido. Por lo tanto, cada espacio de información es una puerta a otros espacios relacionados, los cuales aportan información complementaria o ampliada. El resultado final son complejas estructuras que presenta el conocimiento de un modo que podríamos calificar como tridimensional, con distintas capas de información y variedad en los tipos de contenido.

Estas dos características, el hipermedia y el hipertexto, están generando cambios en el modo no sólo de presentar la información, sino también en el modo en que la leemos, incorporamos y comprendemos.

16. Sitio Web del proyecto: <www.archivodelafrontera.com>.

17. Profesor Titular del Departamento de Historia II de la Universidad de Alcalá.

18. Estos estudios se realizaron durante el Proceso de Boloña con la finalidad de analizar los planes de estudio de las licenciaturas de cada uno de los Estados miembros, la percepción de los empleadores en cuanto a la realidad del mercado laboral y el conocimiento de los graduados y conocer la opinión de profesores y alumnos en cuanto a la formación recibida.

19. Para mayor información consultar C. P. Snow, The Scientific Revolution [en línea], New York, Cambridge University Press, 1961.

20. A. Ruiz Zúñiga, «Consideraciones histórico-filosóficas sobre la tecnología» [en línea], Revista de Filosofía de la Universidad de Costa Rica, n. ${ }^{\circ}$ 59, 1986, p. 28. 


\section{RESÚMENES}

¿Es nuestro sistema educativo anacrónico? ¿Cuáles son las nuevas competencias que requiere la sociedad actual? ¿Está cambiando la tecnología la forma en la que aprendemos? ¿Es posible que las Tecnologías de la Información y la Comunicación nos ayuden en el desarrollo del pensamiento crítico y de la creatividad? ¿Cuál es el futuro de las Humanidades en la «Sociedad del Conocimiento»?

Para dar respuestas a estas preguntas es necesario comprender cómo los cambios tecnológicos y económicos producidos en los últimos 50 años han modificado la sociedad actual, donde nuevas competencias y habilidades son requeridas en el mundo profesional.

La revolución tecnológica que venimos experimentando en el último siglo produjo un desarrollo económico que, a su vez, transformó las sociedades actuales, y con ellas las necesidades formativas y educacionales de los ciudadanos. Para poder hacer frente a estas nuevas necesidades y cambios es fundamental que algunas de esas líneas fronterizas no sean continuas; que puedan comunicarse ambos lados a través de un diálogo integrador, que fomente la formación de humanistas tecnólogos en un proceso de convergencia de la cultura científica y la humanista.

Is our education system outdated? What are the new skills required by today's society? Is technology changing the way we learn? Is it possible that the Information Technology and Communication assist us in the development of critical thinking and creativity? What is the future of the humanities in the "Knowledge Society"?

To provide answers to these questions it is necessary to understand how technological and economic changes produced in the last 50 years have changed the society, where new skills and abilities are required in the professional world.

The technological revolution that we have been experiencing in the last century produced an economic development that, in turn, transformed societies, and with them the training and educational needs of the citizens. To able to cope with these new requirements and changes is essential that some of the border lines are not continuous, that both sides can communicate through an inclusive dialogue that encourages humanistic training technologists in the process of convergence of scientific culture and humanist.

\section{ÍNDICE}

Palabras claves: TIC, educación, humanidades, archivo de la Frontera, Juego del Legajo Keywords: ICT, Education, Humanities, archivo de la Frontera, Juego del Legajo

\section{AUTOR}

\section{LAURA MASSIMINO AMORESANO}

Licenciada en Humanidades, DEA en Modelos Didácticos, Interculturalidad y Aplicaciones de las Nuevas Tecnologías en las Instituciones educativas, Centro Europeo para la Difusión de las Ciencias Sociales 